EPJ Web of Conferences 58, 01011 (2013)

DOI: $10.1051 /$ epjconf/20135801011

(C) Owned by the authors, published by EDP Sciences, 2013

\title{
A New Perspective on Path Integral Quantum Mechanics in Curved Space-Time
}

\author{
Dinesh Singh ${ }^{1,2, a}$ and Nader Mobed ${ }^{1, b}$ \\ ${ }^{1}$ Department of Physics, University of Regina - Regina, Saskatchewan, S4S OA2, Canada \\ ${ }^{2}$ Department of Physics and Engineering Physics, University of Saskatchewan - Saskatoon, Saskatchewan, \\ S7N 5E2, Canada
}

\begin{abstract}
A new approach to path integral quantum mechanics in curved space-time is presented for scalar particle propagation, expressed in terms of Lie transport and Fermi or Riemann normal co-ordinates to describe local curvature. While the presence of local curvature results in a strictly non-unitary representation of local time translation, the formalism nevertheless correctly recovers the free-particle Lagrangian in curved space-time, along with new terms that predict a simultaneous breakdown of time-reversal symmetry and a quantum violation of the weak equivalence principle at the particle's Compton wavelength scale. Furthermore, the formalism reveals the prediction of a gauge-invariant phase factor interpreted as the gravitational Aharonov-Bohm effect and Berry's phase.
\end{abstract}

\section{Introduction}

The Feynman path integral is arguably one of the most elegant and physically intuitive approaches available for describing quantum mechanics, primarily because it provides a single conceptual and mathematical framework for representing classical and quantum phenomena within a unified context [1]. In the on-going search for a viable theory of quantum gravity, the path integral approach as a "sum-over-histories" treatment of quantum mechanics for all possible classical worldlines in spacetime offers a compelling avenue towards reaching this goal. Much of the existing literature on this subject follows from DeWitt's initial treatment decades ago [2], which operates in terms of representing the gravitational field as extrinsic curvature hypersurfaces that are orthogonal to an external time parameter. The path integral representation of quantum field theory in curved space-time [3] also offers some interesting results to fuel thinking in this direction.

Despite the reported successes over the decades, many conceptual and technical challenges still remain that deserve careful consideration. For example, does the curved space-time manifold have a sufficiently smooth structure when making use of the path integral approach? Can the sum-overhistories of all intermediate classical paths be taken consistently without accounting for any forces or related backreaction effects when identifying paths that significantly deviate away from a classical geodesic? Does the correct formulation of path integral quantum mechanics in curved space-time necessarily require the incorporation of space-time torsion $[4,5]$ ? Is it physically permissible to

\footnotetext{
a e-mail: dinesh.singh@uregina.ca

be-mail: nader.mobed@uregina.ca
} 


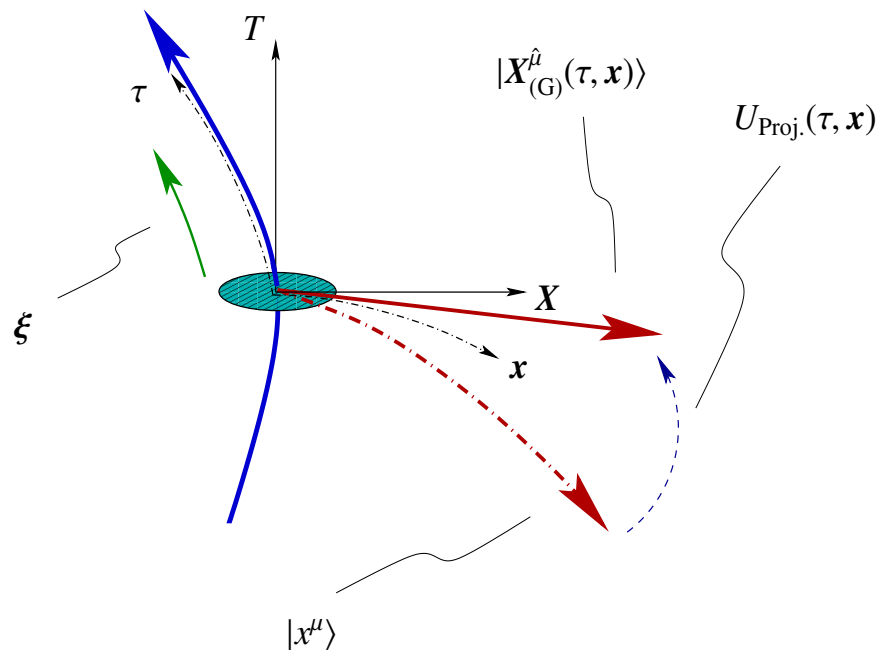

Figure 1. A projection operator $U_{\operatorname{Proj}} .(\tau, \boldsymbol{x})$ is used to transform the position ket vector $\left|x^{\mu}\right\rangle$, defined with respect to Fermi or Riemann normal co-ordinates, into $\left|\boldsymbol{X}_{(\mathrm{G})}^{\hat{\mu}}(\tau, \boldsymbol{x})\right\rangle=U_{\text {Proj. }}(\tau, \boldsymbol{x})\left|x^{\mu}\right\rangle$ that exists on a local tangent space defined at $\tau$. Proper time translation of $\left|\boldsymbol{X}_{(\mathrm{G})}^{\hat{\mu}}(\tau, \boldsymbol{x})\right\rangle$ is determined according to Lie transport with respect to a vector field $\xi$ that is tangent to the reference worldline.

allow the intermediate classical paths to violate causality at a local level, including the possibility to propagate backwards in time as part of the process [6]? At what point is it appropriate to identify a strictly coarse-grained skeletonized form of the path integral with a truly continuum limit? It seems unclear whether any of these questions can be readily answered without any physical data to justify some of the implicit claims made in the literature.

For this paper, a fundamentally different perspective regarding path integral quantum mechanics in curved space-time [7] is presented by considering the propagation of a scalar particle in the presence of intrinsic curvature represented by local Fermi or Riemann normal co-ordinates, in which the sum-over-histories is reflective of Feynman's original perspective in flat space-time [8,9]. Normal co-ordinates are used to incorporate the local flatness theorem in representing space-time curvature as small deviations away from flat space-time, ensuring that the gravitational interactions with quantum matter are locally determined. In what follows, not only is the free-particle Lagrangian in curved space-time recovered-though with quantum-induced fluctuations about the classical space-time metric, important predictions not previously considered within the literature are also presented, namely the breakdown of both time-reversal invariance and the weak equivalence principle at the scalar particle's Compton wavelength scale, some 20 orders of magnitude larger than the Planck scale [7]. The curvature conventions of Misner, Thorne, and Wheeler [10] are assumed throughout this paper, with $c=1$.

\section{Formalism}

To begin [7], consider Fermi or Riemann normal co-ordinates $x^{\mu}=(\tau, \boldsymbol{x}(\tau))$ defined with respect to some local co-ordinate frame, with $\tau$ as some internal time related to a reference time-like worldline $\xi^{\mu}$ within a space-time manifold with metric $g_{\mu \nu}$. It is possible to identify $x^{\mu}$ with a corresponding Hilbert space ket vector $\left|x^{\mu}\right\rangle=|(\tau, \boldsymbol{x})\rangle$. Supposing that $\bar{e}^{\hat{\mu}}{ }_{v}$ is an orthonormal tetrad with hatted indices 
that correspond to a local Lorentz frame with metric $\eta_{\hat{\mu} \hat{v}}$ coinciding with the tangent space, then it follows that $\boldsymbol{X}_{(\mathrm{G})}^{\hat{\mu}}(\tau, \boldsymbol{x})=\bar{e}^{\hat{\mu}_{v}} x^{v}$ with $\eta_{\hat{\alpha} \hat{\beta}} \boldsymbol{X}_{(\mathrm{G})}^{\hat{\alpha}} \boldsymbol{X}_{(\mathrm{G})}^{\hat{\beta}}=g_{\mu v} x^{\mu} x^{v}$ leads to the local Lorentz frame position ket vector $\left|\boldsymbol{X}_{(\mathrm{G})}^{\hat{\mu}}(\tau, \boldsymbol{x})\right\rangle=\left|\bar{e}^{\hat{\mu}}{ }_{v} x^{\nu}\right\rangle$.

For future reference, all indices are identified with the local Lorentz frame and are unhatted. A curvature-dependent local time translation operator $U_{\Delta \tau}\left(V^{\alpha}, \xi^{\alpha}\right)$ can be found [7] in terms of $V^{\mu}=$ $\Delta x^{\mu} / \Delta \tau$ and the Lie derivative $\left(\mathcal{L}_{\boldsymbol{\xi}} \boldsymbol{X}\right)^{\mu}$ along $\boldsymbol{\xi}^{\mu}$, such that

$$
\left|\boldsymbol{X}_{(\mathrm{G})}^{\prime \mu}(\tau+\Delta \tau, \boldsymbol{x}+\Delta \boldsymbol{x})\right\rangle=U_{\Delta \tau}\left|\boldsymbol{X}_{(\mathrm{G})}^{\mu}(\tau, \boldsymbol{x})\right\rangle,
$$

where $\tau$ becomes the proper time for $V^{0}=1$. It is important to note that $U_{\Delta \tau}$ is strictly not unitary because of the presence of local curvature to break this symmetry, in that

$$
U_{\Delta \tau}^{-1}\left(V^{\alpha}, \xi^{\alpha}\right)=U_{-\Delta \tau}\left(V^{\alpha}, \xi^{\alpha}\right) \neq U_{\Delta \tau}^{\dagger}\left(V^{\alpha}, \xi^{\alpha}\right) .
$$

However, this by itself is not inherently problematic, as this formalism remains consistent with Feynman's original derivation in the limit as local space-time curvature vanishes. Indeed, it is this nonunitarity that provides the source for all the interesting predictions to follow.

\section{Configuration Space Path Integral in Curved Space-Time}

Given the initial and final position ket vectors $\left|\boldsymbol{X}_{(\mathrm{i})}^{(\mathrm{G})}\left(\tau_{\mathrm{i}}, \boldsymbol{x}_{\mathrm{i}}\right)\right\rangle$ and $\left|\boldsymbol{X}_{(\mathrm{f})}^{(\mathrm{G})}\left(\tau_{\mathrm{f}}, \boldsymbol{x}_{\mathrm{f}}\right)\right\rangle=$ $U_{\left(\tau_{\mathrm{f}}-\tau_{\mathrm{i}}\right)}^{-1}\left(V^{\alpha}, \boldsymbol{\xi}^{\alpha}\right)\left|\boldsymbol{X}_{(\mathrm{f})}^{(\mathrm{G})}\left(\tau_{\mathrm{i}}, \boldsymbol{x}_{\mathrm{i}}\right)\right\rangle$, where $\tau_{\mathrm{f}}-\tau_{\mathrm{i}}=N \Delta \tau$ for integer $N$, the scalar particle propagator is expressed as

$$
\begin{aligned}
& \left\langle\boldsymbol{X}_{(\mathrm{f})}^{\prime(\mathrm{G})}\left(\tau_{\mathrm{f}}, \boldsymbol{x}_{\mathrm{f}}\right) \mid \boldsymbol{X}_{(\mathrm{i})}^{(\mathrm{G})}\left(\tau_{\mathrm{i}}, \boldsymbol{x}_{\mathrm{i}}\right)\right\rangle \\
& \quad=\left\langle\boldsymbol{X}_{(\mathrm{f})}^{(\mathrm{G})}\left(\tau_{\mathrm{i}}, \boldsymbol{x}_{\mathrm{i}}\right)\left|\left(\prod_{n=1}^{N} \mathbf{1}_{(n)} U_{\Delta \tau}^{-1 \dagger}\right) \mathbf{1}_{(0)}\right| \boldsymbol{X}_{(\mathrm{i})}^{(\mathrm{G})}\left(\tau_{\mathrm{i}}, \boldsymbol{x}_{\mathrm{i}}\right)\right\rangle,
\end{aligned}
$$

where

$$
\mathbf{1}_{(n)}=\int_{-\infty}^{\infty} \mathrm{d}^{3} \boldsymbol{X}_{(n)}\left|\boldsymbol{X}_{(n)}\left(\tau_{n}, \boldsymbol{x}_{n}\right)\right\rangle\left\langle\boldsymbol{X}_{(n)}\left(\tau_{n}, \boldsymbol{x}_{n}\right)\right|
$$

is defined in terms of strictly flat space-time position ket vectors. It is worthwhile to note that, given the Hamiltonian for a free scalar particle as $H(\boldsymbol{P})=\sqrt{\boldsymbol{P} \cdot \boldsymbol{P}+m^{2}}$, where $\boldsymbol{P}$ is the particle's momentum and $m$ is its rest mass, the intermediate momentum states can be integrated out exactly to first-order in the local curvature [7]. This is accomplished by making use of the integral representation of the modified Bessel function $[4,6]$

$$
K_{ \pm v}(\mu \beta)=\frac{\beta^{-v}}{2} e^{-i v \pi / 2} \int_{0}^{\infty} \mathrm{d} N N^{v-1} \exp \left[\frac{i \mu}{2}\left(N-\frac{\beta^{2}}{N}\right)\right]
$$

for $v=1 / 2$, with $\operatorname{Im}(\mu)>0$ and $\operatorname{Im}\left(\mu \beta^{2}\right)<0$, with $\mu$ and $\beta$ appropriately identified in terms of local curvature contributions to the propagator [7].

It eventually follows after integrating over the intermediate momentum states $\boldsymbol{P}_{(n)}$ [7] that

$$
\begin{aligned}
& \left\langle\boldsymbol{X}_{(\mathrm{f})}^{\prime(\mathrm{G})}\left(\tau_{\mathrm{f}}, \boldsymbol{x}_{\mathrm{f}}\right) \mid \boldsymbol{X}_{(\mathrm{i})}^{(\mathrm{G})}\left(\tau_{\mathrm{i}}, \boldsymbol{x}_{\mathrm{i}}\right)\right\rangle=\int \mathcal{D}[\boldsymbol{X}(\tau)] \exp \left[\frac{i}{\hbar} \int_{\tau_{\mathrm{i}}}^{\tau_{\mathrm{f}}} \mathrm{d} \tau L_{(\mathrm{Re}, 0)}^{(\mathrm{G})}\right] \\
& \quad \times \exp \left[\frac{i}{\hbar} \int_{\tau_{\mathrm{i}}}^{\tau_{\mathrm{f}}} \mathrm{d} \tau\left(L_{(\mathrm{Re}, 1)}^{(\mathrm{G})}+i\left[L_{(\mathrm{Im}, 0)}^{(\mathrm{G})}+\lambda \delta(0) L_{(\mathrm{Im}, 1)}^{(\mathrm{G})}\right]\right)\right]
\end{aligned}
$$


where

$$
L_{(\mathrm{Re}, 0)}^{(\mathrm{G})} \approx-m\left[-g_{\mu \nu}^{(\mathrm{eff} .)} \dot{x}^{\mu} \dot{x}^{v}\right]^{1 / 2}
$$

is the expected Lagrangian in curved space-time for a free scalar particle, but now expressed in terms of an effective metric $g_{\mu \nu}^{\text {(eff.) }}$ equal to the classical metric $g_{\mu \nu}$ with quantum fluctuations. The term $L_{(\mathrm{Re}, 1)}^{(\mathrm{G})}$ in (6) can be set to zero by a suitable choice for the Lie derivative terms that help define it. As for $L_{(\mathrm{Im}, 0)}^{(\mathrm{G})}$ and $L_{(\mathrm{Im}, 1)}^{(\mathrm{G})}$ that violate probability due to local curvature, they reveal the prediction of a quantum violation of the weak equivalence principle following from the explicit coupling of curvature with the mass-dependent reduced Compton wavelength $\lambda$, along with a simultaneous prediction of a quantum violation of time reversal invariance. Given that classical gravitation theory is strictly time symmetric in the absence of other considerations, this prediction may suggest an explanation for why time prefers to move forward.

When (6) is expressed in skeletonized form and properly regularized, it is further revealed [7] that a curvature-dependent and gauge-invariant overall phase factor emerges, dependent upon the spatial separation between the initial and final position states. This can be interpreted as a gravitational analogue of the famous Aharonov-Bohm effect [11] and Berry's phase [12], which has the potential to be verified under suitably determined experimental conditions.

\section{Conclusion}

Following Feynman's original vision for implementing path integral quantum mechanics, this paper has demonstrated a fundamentally different approach to incorporate space-time curvature within this framework, compared to other treatments found within the literature. By treating curvature locally in terms of Fermi or Riemann normal co-ordinates, it follows that for a scalar relativistic particle there exists the expected recovery of the free particle Lagrangian in curved space-time, while revealing what seem like significant physical predictions with potentially broad implications for describing quantum mechanical interactions in a non-trivial gravitational field.

It is important to note again that the quantum violation of the weak equivalence principle and time reversal symmetry breakdown predicted within this formalism appear some twenty orders of magnitude larger than the Planck scale, which make them conceivably available for experimental analysis. The fact that only the probability non-conserving contributions to the propagator make this possible suggests that quantum mechanics alone may determine the preferred arrow of time. This indicates the significant potential for identifying fundamental relationships between quantum matter and space-time curvature that would otherwise be obscured from view by adopting a strictly unitary time translation perspective. Furthermore, there exists the prediction of a curvature-dependent and gauge-invariant phase factor identified as the gravitational analogue of the Aharonov-Bohm effect and Berry's phase, worthy of a deeper investigation to probe its physical consequences.

It is worthwhile to consider further developments of this approach when applied to non-zero integer and half-integer spin particles [13]. The results derived from this treatment may provide the foundation for later considering a many-body particle treatment in curved space-time, ultimately leading towards an alternative approach to quantum field theory in curved space-time that may be compared with established approaches contained within the literature [3].

\section{References}

[1] J.J. Sakurai, Modern Quantum Mechanics: Revised Edition (Addison-Wesley, Don Mills, 1994). 
[2] B.S. DeWitt, Rev. Mod. Phys. 29, 377 (1957).

[3] L. Parker and D. Toms, Quantum Field Theory in Curved Spacetime: Quantized Fields and Gravity (Cambridge, New York, 2009).

[4] H. Kleinert, Path Integrals in Quantum Mechanics, Statistics, Polymer Physics, and Financial Markets, fifth ed. (World Scientific, 2009).

[5] H. Kleinert, Mod. Phys. Lett. A 24, 2329 (1989).

[6] R. Ferraro, Phys. Rev. D 45, 1198 (1992).

[7] D. Singh and N. Mobed, Mod. Phys. Lett. A 27, 1250065 (2012).

[8] R.P. Feynman, Rev. Mod. Phys. 20, 367 (1948).

[9] R.P. Feynman and A.R. Hibbs, Quantum Mechanics and Path Integrals (McGraw-Hill, New York, 1965).

[10] C.S. Misner, K.S. Thorne, and J.A. Wheeler, Gravitation (W.H. Freeman \& Co., San Francisco, 1973).

[11] Y. Aharonov and D. Bohm, Phys. Rev. 115, 485 (1959); 123, 1511 (1961).

[12] M.V. Berry, Proc. Roy. Soc. Lond. A 392, 45 (1984).

[13] D. Singh and N. Mobed, work in progress. 
